\title{
Conventional insulin pump therapy in two neonatal diabetes patients harboring the homozygous PTF1A enhancer mutation: Need for a novel approach for the management of neonatal diabetes
}

\author{
Erdal Kurnaz, Zehra Aycan, Nurdan Yıldırım, Semra Çetinkaya \\ Clinic of Pediatric Endocrinology, Dr. Sami Ulus Obstetrics and Gynecology and Pediatrics Training and Research Hospital, \\ Ankara, Turkey. E-mail: erdalkurnaz44@gmail.com \\ Received: 22th December 2016, Revised: 17th March 2017, Accepted: 20th March 2017
}

\begin{abstract}
SUMMARY: Kurnaz E, Aycan Z, Yıldırım N, Çetinkaya S. Conventional insulin pump therapy in two neonatal diabetes patients harboring the homozygous PTF1A enhancer mutation: Need for a novel approach for the management of neonatal diabetes. Turk J Pediatr 2017; 59: 458-462.

The enhancer of PTF1A mutation causes developmental defects of the pancreas. This condition can result in insulin-requiring diabetes and exocrine pancreatic insufficiency. We report two patients with diabetes mellitus harboring the homozygous PTF1A enhancer mutation. The patients had hyperglycemia in the first month of life and were started with subcutaneous insulin injections with NPH insulin. When blood glucose (BG) exceeded $250 \mathrm{mg} / \mathrm{dl}$, a conservative dose of rapid-acting insulin was administered to restore BG to the target range. In cases with documented poor control (persistent hypoglycemia and rebound hyperglycemia), it was decided that the baby would benefit from a continuous subcutaneous insulin infusion pump. But our experience shows that wide fluctuations in BG concentrations despite the strict follow-up was probably due to the absence of circulating glucagon. Further treatment options would overcome this problem, especially for children with pancreas agenesis. We could say theoretically that using diluted insulin, a sensor-augmented insulin pump system and single-hormone (insulin alone) and dual-hormone (insulin and glucagon) artificial pancreas systems may mitigate the severity of hypogylcemia.
\end{abstract}

Key words: conventional insulin pump therapy, neonatal diabetes, PTF1A enhancer mutation.

Permanent neonatal diabetes (NDM) is a very rare disease with an estimated incidence of 1 in $160,000-260,000.1,2$ This is most often caused by heterozygous activating mutations in the gene KCNJ11 and ABCC8. ${ }^{3}$ In about half of these mutation anomalies, epilepsy and developmental retardation can be seen. ${ }^{1-3}$ The syndromes associated with NDM constitute no more than $10 \%$ of all forms of NDM. Cases with PTF1A mutations, which is a cause of syndromic diabetes, have pancreatic and cerebellar agenesis, showing neonatal diabetes. The cases also have a dysmorphic appearance with a triangular face, small chin and abnormalities of the extremities, including joint stiffness. Some of them also have had optic hypoplasia/atrophy. ${ }^{2-4}$ Recently, isolated pancreatic agenesis has been shown to occur in the presence of the enhancer PTFA1 mutation. Morever, the phenotypic effects of PTF1A enhancer mutations are different from those due to PTF1A mutations. No additional clinical features are present in these patients. ${ }^{4}$ Both mutations cause neonatal diabetes, but recently the variable phenotype was defined from Turkey in two sisters with enhancer PTFA1 mutation. ${ }^{5}$

In this article, two cases with enhancer PTF1A mutation causing isolated pancreatic agenesis were presented. Our patients had no additional clinical features, consistent with the reported clinical phenotype of isolated pancreatic agenesis with distal enhancer PTF1A mutations. ${ }^{4,6}$ Continuous subcutaneous insulin infusion (CSII) 
pump was applied to one of the cases in the newborn period and the other one in the $9^{\text {th }}$ month. We aimed to discuss the difficulties in managing the treatment and the methods that could solve these problems.

\section{Case Reports}

\section{Case 1}

Our patient was a boy born at 36 weeks gestation by Caesarian section for oligohydramnios. His birth weight was $1,560 \mathrm{~g}$ (<3rd percentile). $\mathrm{He}$ was the third child of the family, born to first-degree cousins. The first child was an 8 -year-old healthy girl. The second child was a 5-year-old healthy girl. The ethnic origin of the family is Turkish.

The baby was hospitalized due to smallness for gestational age and prematurity. Following establishment of oral feeding, his BG levels began to increase on the first day, with blood glucose of $230 \mathrm{mg} / \mathrm{dl}$. C-peptide and insulin levels were very low (C-peptide $<0.1 \mathrm{ng} / \mathrm{ml}$ and insulin $0.78 \mu \mathrm{IU} / \mathrm{ml}$ ). NPH insulin was administered at a dose of $0.5 \mathrm{U}$ 1-2 times a day; however, he did not gain weight and was referred to our hospital for management of BG control at the age of 9 days. At that time, he passed loose and fatty stools 10-12 times a day. A microscopic examination of the feces revealed the steatocrit was $4(+)$. Genetic analysis was applied to evaluate the etiologic aspect of the case. Serum amylase was low, islet cell, insulin and GAD antibodies were negative (Table I).

Pancreatic tissue could not be shown with USG in the case. A mixture of pancreatic enzymes (Creon ${ }^{\circledR}$ ) was started. The NPH insulin dose was increased to $0.7-1 \mathrm{U} / \mathrm{kg}$ two times a day. When the BG exceeded $250 \mathrm{mg} /$ $\mathrm{dl}$, a conservative dose of rapid-acting insulin was administered to restore BG to the target range. At the age of 1 month, it was difficult to manage this infant because of wide fluctuations in blood glucose concentrations from 50 to $400 \mathrm{mg} / \mathrm{dl}$. As benefits have been shown in cases with documented poor control (persistent hypoglycemia and rebound hyperglycemia), it was decided that the baby would benefit from a CSII pump and continuous glucose monitoring (CBGM). Since CBGM could not be provided, only the most basic component of this system, CSII without CBGM was used (CBGM could not be provided). Blood sugar measurements were made by subcutaneous method. Despite appropriate insulin therapy, hypoglycemia was not prevented. This situation suggests that frequent hypoglycemia could be glucagon deficiency due to pancreatic agenesis.

At his last visit, he was 1.4 years old, weighing $10 \mathrm{~kg}$ (10-25th percentile), and his height was $74 \mathrm{~cm}$ (<3rd percentile). He is currently on 0.69 $\mathrm{U} / \mathrm{kg}$ of insulin per day with CSII, pancreatic enzymes (Creon ${ }^{\circledR} 2500$ lipase U/kg of body weight per meal) and supplementation of fat soluble vitamins. The latest Hbalc was $10.5 \%$. Patient was admitted for insulin dose adjustment. CBGM inserted during hospitalization, and the output of sensor data and duration distribution of glucose levels is given in Figs. 1 and 2. The median percentage time spent at glucose levels below $70 \mathrm{mg} / \mathrm{dl}$ was $17 \%$.

\section{Case 2}

Our patient was a boy born at 38 weeks gestation by Caesarian section for anhydramnios and placenta previa. His birth weight was 1,400 g ( $<3$ rd percentile). He was the fourth child of the family, born to second-degree cousins. The first child was a 14-year-old healthy boy. The second child was a 10-year-old healthy girl. The third child was a 7-year-old healthy girl. The ethnic origin of the family is Turkish.

The baby was hospitalized due to low APGAR scores. Following establishment of oral feeding, his BG levels began to increase with blood glucose of $257 \mathrm{mg} / \mathrm{dl}$. C-peptide and insulin levels were very low (C-peptide $<0.1 \mathrm{ng} / \mathrm{ml}$ and insulin $<2 \mu \mathrm{IU} / \mathrm{ml}$ ). NPH insulin was administered at a dose of 0.5 U 1-2 times a day. He was referred to our hospital for management of BG control at the age of 20 days. At that time, he passed loose and fatty stools 8-10 times a day. A microscopic examination of the feces revealed the steatocrit was $4(+)$. Islet cell, insulin and GAD antibodies were negative (Table I).

Pancreatic tissue could not be shown with USG in the case. A mixture of pancreatic enzymes (Creon ${ }^{\circledR}$ ) was started. The NPH insulin dose was increased to $0.7-1 \mathrm{U} / \mathrm{kg}$ two times a day. At the age of 9 months, his Hbalc was $10.7 \%$ and his wide fluctuations in BG concentrations were from 40 to $500 \mathrm{mg} / \mathrm{dl}$. It was decided that the baby would benefit from a CSII pump and CBGM. As in the first case, only the most basic component of this system, CSII without CBGM was used (CBGM could not be provided). Blood 
Table I. Laboratory Findings of Two Cases with a PTF1A Enhancer Mutation.

\begin{tabular}{lcc}
\hline & Case 1 & Case 2 \\
\hline Blood glucose, mg/dl & 278 & 305 \\
Simultaneous insulin, $\mu \mathrm{IU} / \mathrm{ml}$ (normal: 2-23) & $<2$ & $<2$ \\
Simultaneous C-peptide, ng/ml (normal: $0.9-4)$ & $<0.2$ & $<0.2$ \\
Islet cell antibody, U/ml (normal: <1) & 0.63 & 0.55 \\
GAD antibody, IU/ml (normal: 0-10) & 0.68 & 0.13 \\
Insulin antibody, \% (normal: 0-7) & 3.86 & 4.77 \\
Amylase, U/l (normal: 25-125) & 22 & - \\
\hline
\end{tabular}

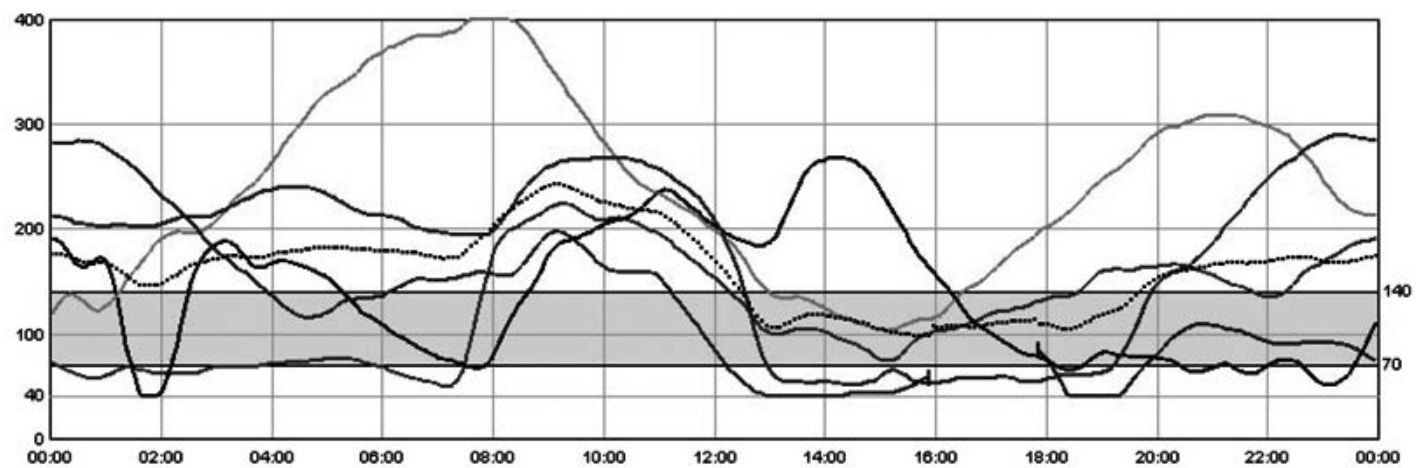

Fig. 1. Continuous glucose monitoring sensor data over 6 days (Case 1). Wide fluctuations in blood glucose concentrations despite the strict follow up.
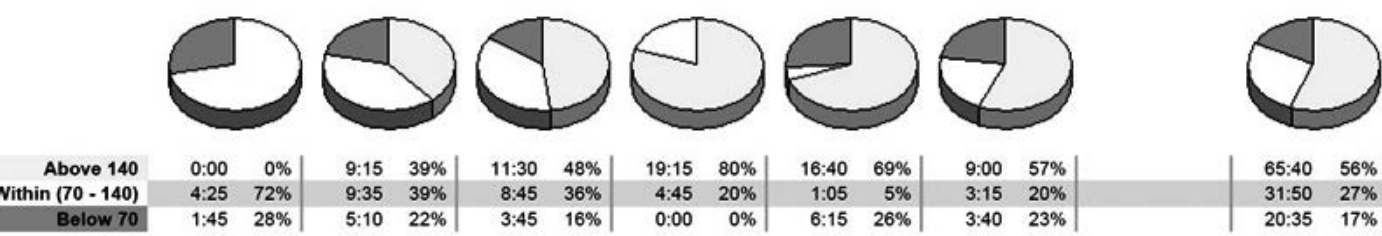

\begin{tabular}{rr|rr|}
$16: 40$ & $69 \%$ & $9: 00$ & $57 \%$ \\
$1: 05$ & $5 \%$ & $3: 15$ & $20 \%$
\end{tabular}

\begin{tabular}{rr|rr|}
$1: 05$ & $5 \%$ & $3: 15$ & $20 \%$ \\
\hline $6: 15$ & $26 \%$ & $3: 40$ & $23 \%$
\end{tabular}

$65: 40 \quad 56 \%$

Fig. 2. Duration distribution of glucose levels (Case 1).

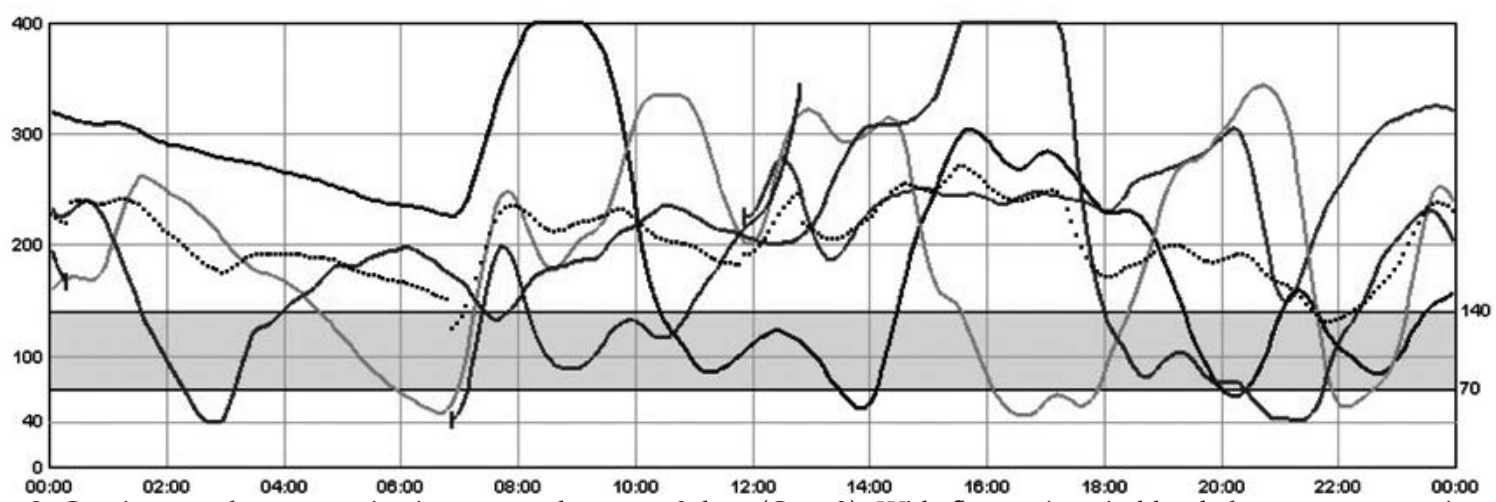

Fig. 3. Continuous glucose monitoring sensor data over 6 days (Case 2). Wide fluctuations in blood glucose concentrations despite the strict follow up.
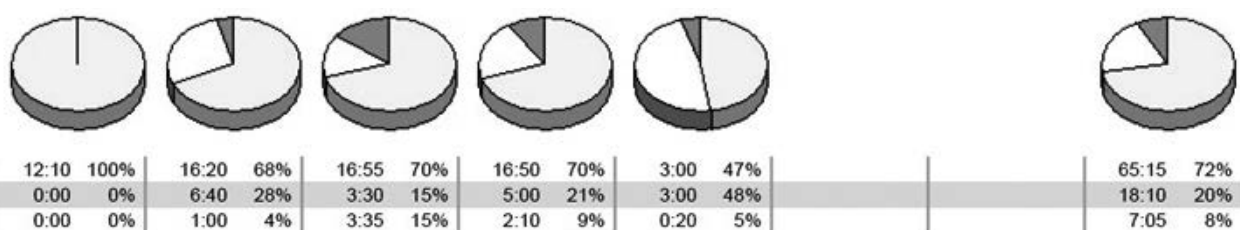

\begin{tabular}{ll|ll|}
$16: 50$ & $70 \%$ & $3: 00$ & $47 \%$
\end{tabular}

\begin{tabular}{|rr|rr|}
\hline $5: 00$ & $21 \%$ & 3.00 & $48 \%$ \\
\hline $2: 10$ & $9 \%$ & $0: 20$ & $5 \%$
\end{tabular}

Fig. 4. Duration distribution of glucose levels (Case 2). 
sugar measurements were made by subcutaneous method. Despite appropriate insulin therapy, hypoglycemia was not prevented. This situation suggests that frequent hypoglycemia could be glucagon deficiency due to pancreatic agenesis.

At his last visit, he was 3.5 years old, weighing $14.8 \mathrm{~kg}$ (25th percentile), and his height was $97.4 \mathrm{~cm}$ (25th percentile). He is currently on $0.6 \mathrm{U} / \mathrm{kg}$ of insulin per day with CSII, pancreatic enzymes (Creon 1000 lipase U/kg of body weight per meal) and supplementation of fat soluble vitamins. The latest Hbalc was $8 \%$. Patient admitted for insulin dose adjustment. CBGM inserted during hospitalization, and the output of sensor data and duration distribution of glucose levels is given in Figs. 3 and 4. The median percentage of time spent at glucose levels below $70 \mathrm{mg} / \mathrm{dl}$ was $8 \%$.

\section{Genetic Analysis}

Sanger sequencing of the PTF1A enhancer in these patients identified a homozygous mutation, g.23508437A $>$ G. The parents were heterozygous for the same mutation. No other family members were affected.

Written informed consent was obtained from the parents.

\section{Discussion}

We describe two patients who were born to different consanguineous families of Turkish origin who had a homozygous mutation in a described distal PTF1A enhancer. They had severe insulin and exocrine pancreatic deficiency. Treatment with NPH insulin and a mixture of pancreatic enzymes (Creon $\left.{ }^{\circledR}\right)$ was introduced. Due to wide fluctuations in BG concentrations despite strict follow-up, the patients were started on management with CSII. Optimal levels of HbAlc could not be achieved despite the close follow-up of the patients. High blood sugar after frequent hypoglycemia intervention may have caused this condition.

Good glycemic control is important, since hypoglycemia, as well as hyperglycemia, have been linked to adverse neurological outcomes. ${ }^{7-13}$ The combination of CSII and CBGM has allowed detailed data to be obtained on glucose control in patients with diabetes, and especially in very young children and adolescents its use has increased remarkably over the last years in many countries. Large numbers of clinical studies have established that CSII in pediatrics is safe and effective. ${ }^{10,11}$ This experience appears to be similar to others who have also managed infants with neonatal diabetes. ${ }^{6-9}$ It has been difficult to manage these infants with CSII because of inconsistency of appetite and feeding schedule, malabsorption, subcutaneous infections at the pump site insertion, and frequent illnesses.

Pancreatic agenesis is defined as pancreatic betacell failure indicated by neonatal diabetes requiring insulin treatment and exocrine pancreatic insufficiency requiring enzyme replacement therapy. ${ }^{4}$ On the other hand, it was reported that a patient with pancreatic agenesis had an absence of circulating glucagon immunoreactivity. ${ }^{14}$ In health, insulin and glucagon exert opposing effects on glucose homeostasis by suppressing or stimulating the hepatic glucose output that depends on the balance between them. It has been demonstrated that a low amount of $\alpha$-cell ( $2 \%$ of the normal $\alpha$-cell mass) is necessary to prevent the metabolic dysregulation observed in diabetes. ${ }^{16}$ So unsurprisingly hypoglycemia (especially resistant) was very common during CSII treatment in our case, probably due to absence of circulating glucagon.

Theoretically, administration of basal longacting insulins (e.g., insulin glargine) would overcome this problem, however there have been no pharmacokinetic studies in these kinds of patients. On the other hand, the need for insulin may be low during the infant and neonatal period. Some children require as little as $0.2-0.3$ $\mathrm{U}$ of bolus insulin. These factors pose a challenge for precise and stable continuous subcutaneous insulin delivery as well as for occlusion alarm triggering in insulin pumps. ${ }^{17}$ Therefore, the production of low-dose insulin systems or less concentrated insulins is particularly important for children in the first years of life. Another practical step is a combination of CSII pump and CBGM. The low glucose suspend feature of the sensor augmented insulin pump can automatically stop insulin delivery when a predetermined glucose threshold is reached. Danne et al. ${ }^{16}$ and Garg et al. ${ }^{18}$ showed that this pump prevents hypoglycemia. A more elaborate system is the so-called artificial pancreas, which titrates insulin delivery according to glucose sensor readings.

Recent efforts have 'resurrected' glucagon as a key hormone in the pathophysiology of diabetes. New studies target its abnormal regulation and action that is key for improving diabetes treatment. Haidar et al. ${ }^{19}$ reported that insulin 
and glucagon closed-loop delivery for controlling glucose levels in adults with type 1 diabetes improved short-term glucose control and reduced the risk of hypoglycemia in a group of 15 adults with type 1 diabetes. Another study showed that the single-hormone (insulin alone) and dual-hormone (insulin and glucagon) artificial pancreas systems both provided better glycemic control than did conventional insulin pump therapy. ${ }^{20}$ We consulted to be the first center to obtain a dual pump, but did not achieve this due to transfer and hospitalization problems. Currently, it may not be possible to routinely use a dual (insulin and glucagon) pump due to technical problems, but it can give promise for our patients.

A different perspective is that the counterregulatory hormone, glucagon, can provide replacement therapy for diabetes and may improve glucose and insulin levels and prevent hypoglycemia, but in addition may have several unintended consequences that could further compromise the regulatory response to an altered metabolic state. Alternatively, using diluted insulin, using sensor-augmented insulin pump therapy, a single-hormone (insulin alone) artificial pancreas system can be used instead of a dual-hormone system (insulin and glucagon), especially with pancreas agenesia causing persistent hypoglycemia.

\section{REFERENCES}

1. Wiedemann B, Schober E, Waldhoer T, et al. Incidence of neonatal diabetes in Austria - calculation based on the Austrian Diabetes Register. Pediatr Diabetes 2010; 11: 18-23.

2. Slingerland AS, Shields BM, Flanagan SE, et al. Referral rates for diagnostic testing support an incidence of permanent neonatal diabetes in three European countries of at least 1 in 260,000 live births. Diabetologia 2009; 52: 1683-1685.

3. Rubio-Cabezas O, Ellard S. Diabetes mellitus in neonates and infants: Genetic heterogeneity, clinical approach to diagnosis, and therapeutic options. Horm Res Paediatr 2013; 80: 137-146.

4. Weedon MN, Cebola I, Patch AM, et al; International Pancreatic Agenesis Consortium. Recessive mutations in a distal PTF1A enhancer cause isolated pancreatic agenesis. Nat Genet 2014; 46: 61-64.

5. Gonc EN, Ozon A, Alikasifoglu A, et al. Variable phenotype of diabetes mellitus in siblings with a homozygous PTF1A enhancer mutation. Horm Res Paediatr 2015; 84: 206-211.

6. Demirbilek H, Arya VB, Ozbek MN, et al. Clinical characteristics and molecular genetic analysis of 22 patients with neonatal diabetes from the South-Eastern region of Turkey: Predominance of non-KATP channel mutations. Eur J Endocrinol 2015; 172: 697-705.

7. Thomas IH, Saini NK, Adhikari A, et al. Neonatal diabetes mellitus with pancreatic agenesis in an infant with homozygous IPF-1 Pro63fsX60 mutation. Pediatr Diabetes 2009; 10: 492-496.

8. Bharucha T, Brown J, McDonnell C, et al. Neonatal diabetes mellitus: Insulin pump as an alternative management strategy. J Paediatr Child Health 2005; 41: $522-526$

9. Wintergerst KA, Hargadon S, Hsiang HY. Continuous subcutaneous insulin infusion in neonatal diabetes mellitus. Pediatr Diabetes 2004; 5: 202-206.

10. Passanisi S, Timpanaro T, Lo Presti D, Mammì C, Caruso-Nicoletti M. Treatment of transient neonatal diabetes mellitus: insulin pump or insulin glargine? Our experience. Diabetes Technol Ther 2014; 16: 880884.

11. Beardsall K, Pesterfield CL, Acerini CL. Neonatal diabetes and insulin pump therapy. Arch Dis Child Fetal Neonatal Ed 2011; 96: F223-F224.

12. Northam EA, Anderson PJ, Jacobs R, Hughes M, Warne GL, Werther GA. Neuropsychological profiles of children with type 1 diabetes 6 years after disease onset. Diabetes Care 2001; 24: 1541-1546.

13. Ferguson SC, Blane A, Wardlaw J, et al. Influence of an early-onset age of type 1 diabetes on cerebral structure and cognitive function. Diabetes Care 2005; 28: 1431-1437.

14. Howard CP, Go VL, Infante AJ, Perrault J, Gerich JE, Haymond MW. Long-term survival in a case of functional pancreatic agenesis. J Pediatr 1980; 97: 786-789.

15. Thorel F, Damond N, Chera S, et al. Normal glucagon signaling and $\beta$-cell function after near-total $\alpha$-cell ablation in adult mice. Diabetes 2011; 60: 2872-2882.

16. Danne T, Kordonouri O, Holder M, et al. Prevention of hypoglycemia by using low glucose suspend function in sensor-augmented pump therapy. Diabetes Technol Ther 2011; 13: 1129-1134.

17. Mianowska B, Szadkowska A, Fendler W, Mlynarski W. Use of lispro insulin diluted with normal saline to $10 \mathrm{U} / \mathrm{ml}$ in an insulin pump: Case report. J Diabetes Sci Technol 2012; 6: 1238-1239.

18. Garg S, Brazg RL, Bailey TS, et al. Reduction in duration of hypoglycemia by automatic suspension of insulin delivery: The in-clinic ASPIRE study. Diabetes Technol Ther 2012; 14: 205-209.

19. Haidar A, Legault L, Dallaire M, et al. Glucoseresponsive insulin and glucagon delivery (dual-hormone artificial pancreas) in adults with type 1 diabetes: A randomized crossover controlled trial. CMAJ 2013; 185: 297-305.

20. Haidar A, Legault L, Messier V, et al. Comparison of dual-hormone artificial pancreas, single-hormone artificial pancreas, and conventional insulin pump therapy for glycemic control in patients with type 1 diabetes: An open-label randomised controlled crossover trial. Lancet Diabetes Endocrinol 2015; 3: $17-26$. 